\title{
A severe case of COVID-19 in an adolescent with PIMS-TS, cardiomyopathy, and pulmonary embolism.
}

\author{
Andreea Panciu ${ }^{1}$, Cristina Neaga ${ }^{1}$, Marina Popov ${ }^{1}$, Adina Ivan $^{1}$, Florica Popa ${ }^{1}$, Anuta \\ Bilasco $^{1}$, Cornelia Dogaru ${ }^{1}$, Camelia Kouris ${ }^{1}$, Cristina Negulescu ${ }^{1}$, Anca Draganescu ${ }^{1}$, \\ Roxana Matei $^{1}$, Gheorghita Jugulete ${ }^{1}$, and Monica Luminos ${ }^{1}$ \\ ${ }^{1}$ Institutul National de Boli Infectioase Prof Dr Matei Bals
}

October 12,2020

\begin{abstract}
We report the case of a 14-year-old male patient with no previous history of cardiovascular disease or thromboembolic episodes, admitted to our hospital with a severe form of COVID-19, in the 9th day of disease evolution, with acute respiratory and heart failure symptoms. Chest computer tomography showed bilateral multilobular ground-glass opacities, consolidations, and a sub segmental arterial branch thromboembolism. The echocardiography showed a dilated left ventricle with severely impaired left ventricular function $(\mathrm{LVEF}=30 \%)$. Blood test showed extremely elevated NT pro-BNP (22 558 ng/L), inflammatory markers and D-dimers. The diagnosis of PIMS-TS, COVID-19 sepsis-related cardiomyopathy and pulmonary thromboembolism was made, with a favourable outcome under supportive treatment. Clinicians should be aware of this severe presentation of COVID-19 in children.
\end{abstract}

\section{Title page}

Complete manuscript title: A severe case of COVID-19 in an adolescent with PIMS-TS, cardiomyopathy, and pulmonary embolism.

Authors names: Andreea Madalina Panciu ${ }^{1}$, Cristina Neaga ${ }^{1}$, Marina Popov$^{1}$, Adina Ivan ${ }^{1}$, Florica Popa $^{1}$, Anuta Bilasco ${ }^{1}$, Cornelia Dogaru ${ }^{1}$, Camelia Kouris ${ }^{1}$, Cristina Negulescu ${ }^{1}$, Anca Draganescu ${ }^{1}$, Roxana Matei $^{1}$, Asst. Prof. Gheorghita Jugulete ${ }^{1,2}$, Prof. Luminita Monica Luminos ${ }^{1,2}$.

Affiliations: 1 - National Institute of Infectious Disease Prof. Dr. Matei Bals, Pediatric Infectious Disease 2 - University of Medicine and Pharmacy Carol Davila Bucharest

\section{Corresponding author:}

Name: Andreea Madalina Panciu

Address: Street Stefan cel Mare nr.21, block C2, ap 34, sector 2, Bucharest, Romania.

Telephone number: +40720114671

E-mail: panciu.andreea.madalina@gmail.com

No source of funding.

Key words: COVID-19, SARS-COV2, sepsis related cardiomyopathy, pulmonary thrombemobolism;

Running head title: PIMS-TS, cardiomyopathy, and PE in an adolescent.

Hosted file 
Manuscript.pdf available at https://authorea.com/users/366396/articles/486180-a-severe-caseof-covid-19-in-an-adolescent-with-pims-ts-cardiomyopathy-and-pulmonary-embolism

\section{Hosted file}

Table 1.pdf available at https://authorea.com/users/366396/articles/486180-a-severe-case-ofcovid-19-in-an-adolescent-with-pims-ts-cardiomyopathy-and-pulmonary-embolism
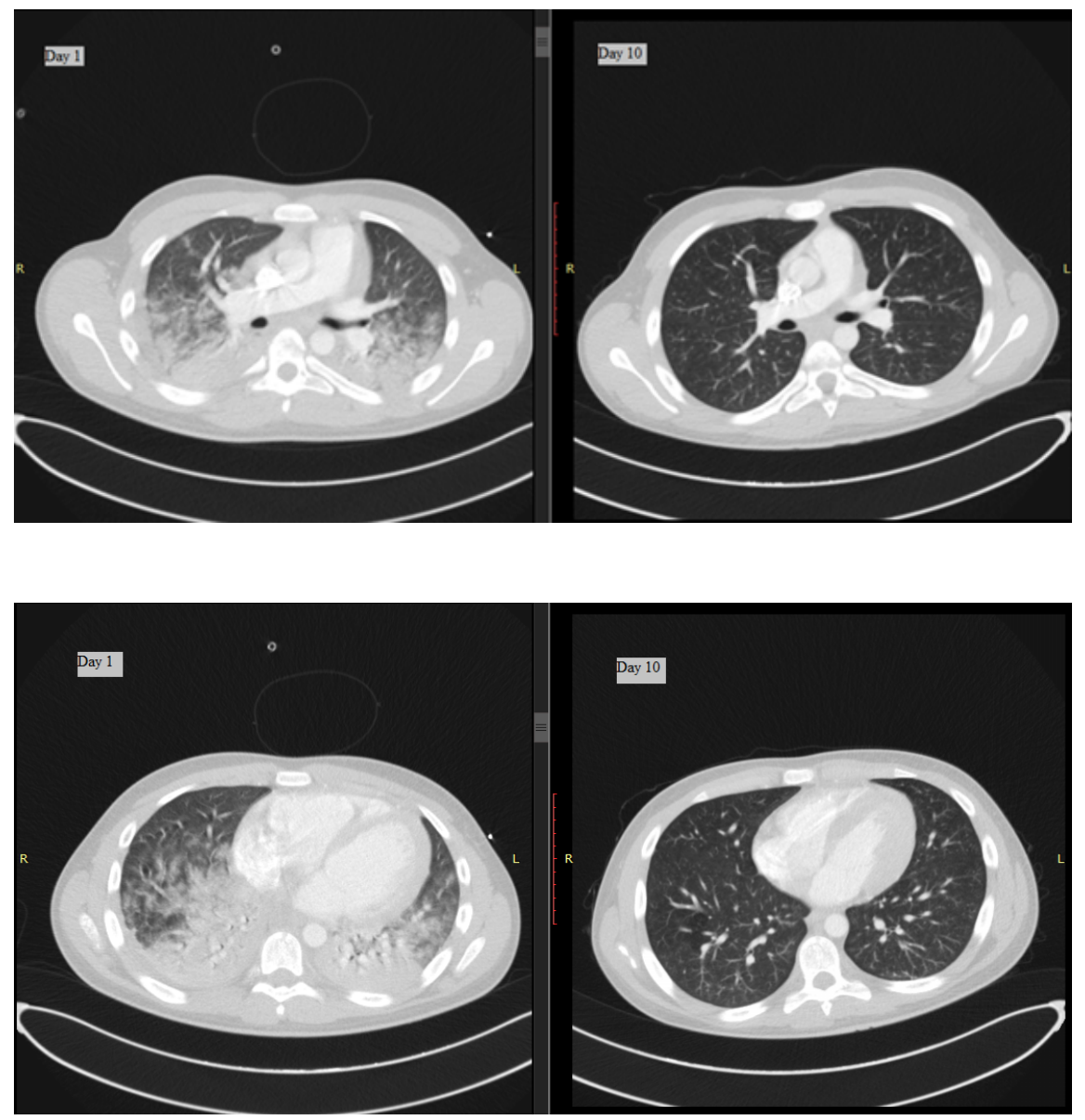